\title{
Finite temperature and the Polyakov loop in the covariant varia- tional approach to Yang-Mills Theory
}

\author{
Markus Quandt ${ }^{1, a}$ and Hugo Reinhardt ${ }^{1, b}$ \\ ${ }^{1}$ Universität Tübingen, Institut für Theoretische Physik, Auf der Morgenstelle 14, D-72076 Tübingen, Ger- \\ many
}

\begin{abstract}
We extend the covariant variational approach for Yang-Mills theory in Landau gauge to non-zero temperatures. Numerical solutions for the thermal propagators are presented and compared to high-precision lattice data. To study the deconfinement phase transition, we adapt the formalism to background gauge and compute the effective action of the Polyakov loop for the colour groups SU(2) and SU(3). Using the zero-temperature propagators as input, all parameters are fixed at $\mathrm{T}=0$ and we find a clear signal for a deconfinement phase transition at finite temperatures, which is second order for $\mathrm{SU}(2)$ and first order for $\mathrm{SU}(3)$. The critical temperatures obtained are in reasonable agreement with lattice data.
\end{abstract}

\section{Introduction}

The low energy sector of quantum chromodynamics (QCD) and, in particular, its phase diagram are among the most actively researched topics in elementary particle physics. While heavy ion collisions at the large hadron collider (LHC) now begin to explore in detail the quark-gluon plasma at large temperatures and baryon densities, the theoretical description of the full phase diagram through lattice simulations is still hampered by the sign problem. Alternative functional methods in the continuum are therefore of particular interest. In covariant gauges, the most widely used tools are functional renormalization group (FRG) flow equations [1] and Dyson-Schwinger equations (DSE) [2], while extensions of the Faddeev-Popov action through mass terms [3] or the Gribov-Zwanziger term [4] are also discussed. If we are willing to dispense with manifest covariance, a particularly appealing and physically transparent picture emerges in the Hamiltonian approach to QCD in Coulomb gauge using variational techniques [5].

Recently, we have proposed an alternative continuum approach [6] which attempts to combine the insightfulness of the Hamiltonian approach with the simplicity of a manifestly covariant setup. The method is based on simple Ansätze for the euclidean path integral measure, and results in a closed set of integral equations that can be conventionally renormalized. The numerical solutions give excellent agreement with zero-temperature lattice propagators [6]. In this talk, I will briefly review the method and its findings at zero temperature, and then present the recent extension to non-zero temperatures [7]. The propagators will turn out to be only mildly affected by temperature, and no clear qualitative

\footnotetext{
a e-mail: markus.quandt@uni-tuebingen.de (speaker)

be-mail: hugo.reinhardt@uni-tuebingen.de
} 
change can be observed across the deconfinement phase transition. The question of confinment must hence be studied by other means. This is discussed in the second part of this talk, where I report on the variational calculation of the effective action for the Polyakov loop (the order parameter for the deconfinement phase transition) at varying temperatures [8]. We find a clear signal for a phase transition which is second order for the colour group SU(2), and first order for SU(3), and obtain critical temperatures $T^{*}$ that are in good agreement with lattice data. Finally, I conclude this talk with a brief summary and an outlook on future developments.

\section{Covariant variational approach to Yang-Mills Theory}

Let us briefly recall the variation principle for the effective action in the quantum theory of a field $A(x)$ in euclidean spacetime. The variation is with respect to the normalized path integral measure $d \mu(A)$ which is used to compute expectation values of arbitrary observables. Within the space of such probability measures, quantum field theory singles out the particular Gibb's type of measure

$$
d \mu_{0}[A]=Z^{-1} \mathcal{D} A \exp \left\{-\hbar^{-1} S[A]\right\}
$$

where $S[A]$ is the classical (euclidean) action, and the partition function $Z$ is required for normalization. The moments of $d \mu_{0}$ are the usual Schwinger functions of euclidean field theory. Moreover, Gibb's measure minimizes the free action

$$
F(\mu) \equiv\langle S[A]\rangle_{\mu}-\hbar \mathcal{W}(\mu)
$$

where the entropy $\mathcal{W}[\mu]=-\langle\ln \rho[A]\rangle_{\mu}$ describes the available phase space for quantum fluctuations in the trial measure $d \mu=\mathcal{D} A \rho[A]$. It is convenient to perform the minimization of the free action in two steps, by first constraining $F(\mu)$ such that the expectation value of an arbitrary operator $\Omega[A]$ is fixed at a prescribed classical value $\omega$. The minimum is then called the effective action for the operator $\Omega$,

$$
\Gamma[\omega]=\min _{\mu}\left\{F(\mu) \mid\langle\Omega\rangle_{\mu}=\omega\right\} .
$$

The most common choice is to take $\Omega$ as the quantum field itself (with classical value $\langle A\rangle_{\mu}=\mathcal{A}$ ) whence the derivatives of $\Gamma[\mathcal{A}]$ become the 1PI proper functions of the full quantum theory. For Yang-Mills theory in the continuum, the exact Gibb's measure has both gauge-fixing terms in the action and a Faddeev-Popov determinant $\mathcal{J}[A]$ in the measure. This modifies the weight of quantum fluctuations (and hence the free action) by replacing the entropy with the so-called relative entropy

$$
\overline{\mathcal{W}}(\mu)=\mathcal{W}(\mu)+\langle\ln \mathcal{J}[A]\rangle_{\mu}=-\langle\ln (\rho / \mathcal{J})\rangle_{\mu} .
$$

Next, we need to make a physically sensible ansatz for the probability measure $d \mu$. The simplest choice is a modified Gaussian of the form

$$
d \mu[A]=\mathcal{N} \cdot \mathcal{D} A \mathcal{J}[A]^{1-2 \alpha} \cdot \exp \left\{-\int d(x, y) A(x) \omega(x, y) A(y)\right\} .
$$

The parameter $\alpha$ and the kernel $\omega$ are variational parameters. Note that the ansatz space (4) is not large enough to cover the exact Gibb's measure, i.e. the restricted variation in the space (4) will give an approximation to the true theory through Gaussian measures, and this approximation is optimal in 
the sense of the effective action. For Yang-Mills theory in Landau gauge, however, we still have to deal with the normalized Faddeev-Popov determinant,

$$
\mathcal{J}[A]=\frac{\operatorname{det}\left(-\partial_{\mu} \hat{D}_{\mu}\right)}{\operatorname{det}\left(-\partial^{2}\right)} .
$$

If we envision a formal loop counting parameter in the exponent of the ansatz (4), it is easy to see that $\mathcal{J}[A]$ can be replaced, to two-loop order in the action, by the simpler expression

$$
\ln \mathcal{J}[A] \approx-\frac{1}{2} \int d(x, y) A_{\mu}^{a}(x) \chi_{\mu \nu}^{a b}(x, y) A_{v}^{b}(y),
$$

where the curvature $\chi$, to the same loop order, can be expressed through the Faddeev-Popov ghost operator $G=\left(\partial_{\mu} \hat{D}^{\mu}\right)^{-1}$ and the bare $\left(\Gamma_{0}\right)$ and full $(\Gamma)$ ghost-gluon vertex,

$$
\chi_{\mu \nu}^{a b}(x, y)=-\left\langle\frac{\delta^{2} \ln \mathcal{J}}{\delta A_{\mu}^{a}(x) \delta A_{\nu}^{b}(y)}\right\rangle=-\operatorname{Tr}\left[\langle G\rangle \Gamma_{\mu}^{a}(x)\langle G\rangle \Gamma_{0, v}^{b}(y)\right] .
$$

By global colour invariance and the Landau gauge condition, we can write $\chi_{\mu \nu}^{a b}(k)=\delta^{a b} t_{\mu \nu}(k) \chi(k)$, where $t_{\mu \nu}(k)$ is the transversal projector in momentum space. For the ghost propagator appearing in eq. (7), we can eventually employ an exact resolvent identity. To the given loop order, this leads to and integral equation for the scalar curvature $\chi(k)$ which can be written symbolically as the ghost loop,

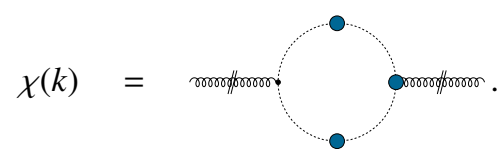

At this point, we have to make the assumption that the full ghost-gluon vertex appearing on the rhs is bare (rainbow approximation). This simplification is expected to be very robust, since the vertex is known to be non-renormalized in Landau gauge due to Taylor's identity [9], and lattice studies indeed indicate that it receives only very mild corrections in the infrared [10]. With this approximation, the ghost form factor $\eta(k) \equiv k^{2}\langle G(k)\rangle$ entering (8) can be written as ${ }^{1}$

$$
\eta(k)^{-1}=1-N g^{2} \int \frac{d^{4} q}{(2 \pi)^{4}} \frac{\eta(k-q)}{(k-q)^{2}} \frac{1-(\hat{k} \cdot \hat{q})}{\omega(k)} .
$$

We can now take the expectation values of both the gauge fixed Yang-Mills action and the relative entropy, and obtain the optimal choice for the variation kernel $\omega$ from the gap equation $\delta F / \delta \omega(k)=0$,

$$
\omega(k)=k^{2}+M^{2}+\chi(k),
$$

where $M^{2}$ is a (quadratically divergent) contstant induced by the tadpole diagram.

The system of integral equations requires renormalization. As layed out in detail in Refs. [6] and [8], we need three counterterms

$$
\mathcal{L}_{\mathrm{CT}}=\delta Z_{A} \cdot \frac{1}{4}\left(\partial_{\mu} A_{v}^{a}-\partial_{v} A_{\mu}^{a}\right)^{2}+\delta M^{2} \cdot \frac{1}{2}\left(A_{\mu}^{a}\right)^{2}+\delta Z_{c} \cdot\left(\partial_{\mu} \eta\right)^{2}
$$

\footnotetext{
${ }^{1}$ The variation kernel only enters in the combination $\bar{\omega}=\omega+(1-2 \alpha) \chi$, which equals the inverse gluon propagator. The value of the variational parameter $\alpha$ is hence immaterial and, for simplicity, we will write $\omega(k)$ instead of $\bar{\omega}(k)$.
} 

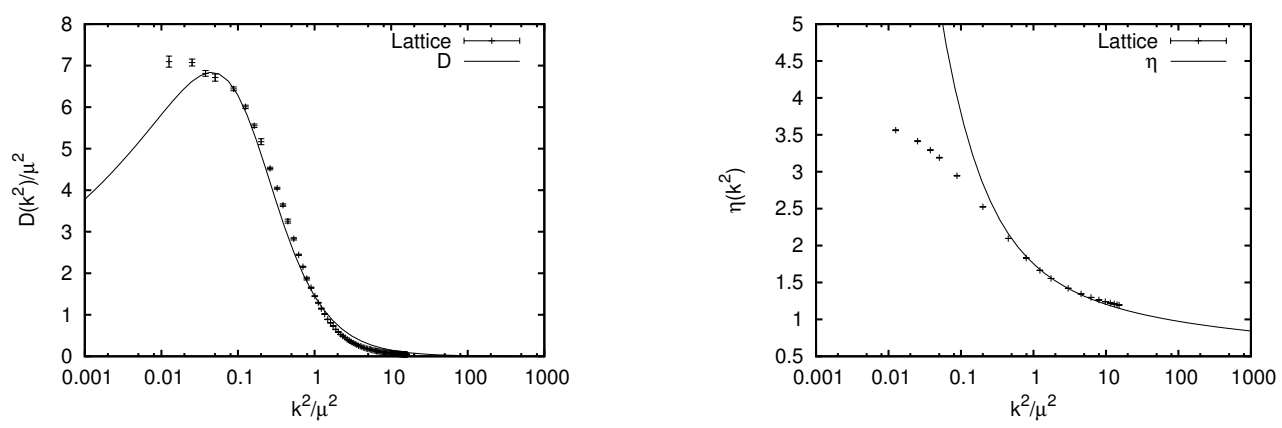

Figure 1. The zero-temperature gluon propagator (left) and the ghost form factor (right) for the scaling type of renormalization condition, compared to high-precision lattice data [11].
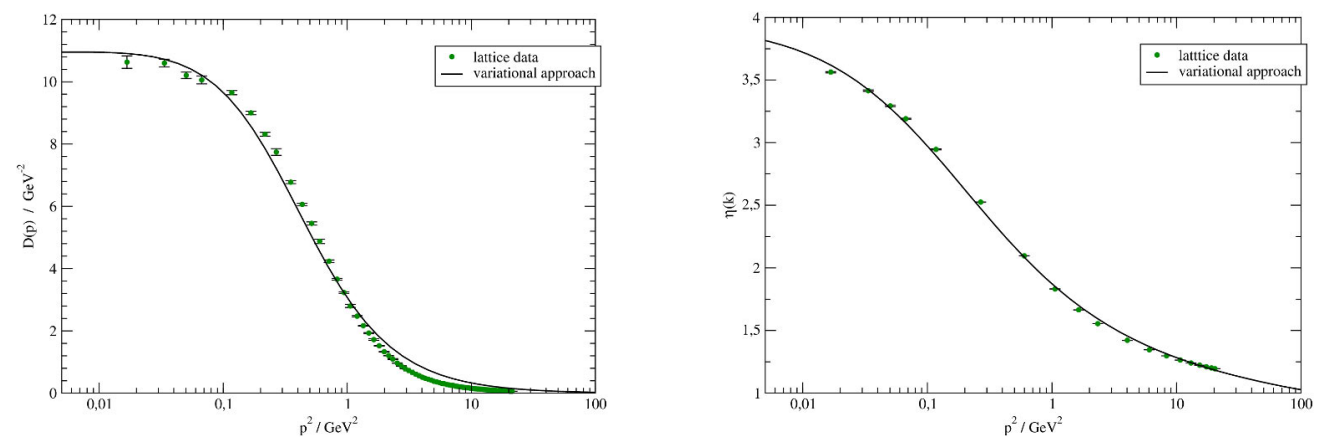

Figure 2. The zero-temperature gluon propagator (left) and the ghost form factor (right) for the decoupling type of renormalization condition, compared to high-precision lattice data [11].

To fix the coefficients, we prescribe the values $Z$ and $M_{A}$ in the conditions $\omega\left(\mu_{0}\right)=Z M_{A}^{2}$ and $\omega(\mu)=$ $Z \mu^{2}$ at two different scales $\mu_{0} \ll \mu$. This removes all quadratic and subleading logarithmic divergences from the gap equation. ${ }^{2}$ In addition, we must also remove the logarithmic divergence in the ghost equation (9), for which we fix of the ghost form factor at $\mu_{c} \rightarrow 0$. The reasoning here is that the present approach allows not only for a single solution, but instead for a whole family of scaling and decoupling solutions, which differ only in their deep infrared behaviour. Fixing the ghost form factor at a small scale thus selects a specific type of solution and avoids numerical instabilities in the deep infrared.

For the scaling type of solution $\left(\eta(0)^{-1}=0\right)$, we find the usual power-law behaviour of the propagators

$$
\omega(k) \sim\left(k^{2}\right)^{\alpha}, \quad \eta(k) \sim\left(k^{2}\right)^{-\beta}
$$

with the same exponents $\alpha \approx 0.191$ and $\beta \approx 0.595$ also observed in the FRG and DSE approach. As can be seen from Fig. 1, the scaling solution gives a rather poor description of the lattice data in the

\footnotetext{
${ }^{2}$ Note that the condition for the "mass counterterm" is not imposed at $\mu_{0}=0$ and $M_{A}^{2}$ hence does not have the meaning of a (constituent) mass. In fact, the mass parameter $M_{A}^{2}$ mainly affects the mid-momentum region and also appears in the renormalization of the scaling type of solution, where no gluon mass emerges.
} 
deep infra-red. By contrast, the decoupling solution $\left(\eta(0)^{-1}>0\right)$ plotted in fig. 2 gives an excellent description of the lattice findings for both the gluon and ghost propagator. We will use the decoupling solution obtained here as input for the Polyakov loop studies in section 4.

\section{Propagators at finite temperature}

Since our variation principle computes the moments (Schwinger functions) from a conventional euclidean quantum field theory, the extension to finite temperature through the imaginary time formalism is straightforward: We compactify the euclidean time direction $x_{0} \in[0, \beta]$ and impose periodic boundary conditions for gluons and ghosts [12]. Momentum integrals over the frequency $k_{0}$ are replaced by Matsubara sums,

$$
\int \frac{d^{4} k}{(2 \pi)^{4}} \cdots \quad \Longrightarrow \quad \int_{\beta} \mathrm{đ} \cdots \equiv \beta^{-1} \sum_{n \in \mathbb{Z}} \int \frac{d^{3} \mathbf{k}}{(2 \pi)^{3}} \cdots
$$

and fields in momentum space are now functions of $\mathbf{k}$ and the Matsubara frequency $k_{0}=v_{n} \equiv 2 \pi n / \beta$ separately. At finite temperatures, the euclidean $O(4)$ invariance is broken because the heat bath singles out a rest frame with direction $v_{\mu}=(1,0,0,0)$. As a consequence, all symmetric rank-2 tensors such as the gluon propagator, the variation kernel $\omega_{\mu \nu}(k)$ and the curvature $\chi_{\mu \nu}(k)$ must be linear combinations of $O(3)$-invariant rank-2 tensors that can be formed from $k_{\mu}$ and $v_{\mu}$. Also taking into account the Landau gauge fixing condition, this leaves us with just $t w o$ remaining Lorentz structures, ${ }^{3}$

$$
\omega_{\mu \nu}^{a b}(k)=\delta^{a b} \omega_{\perp}(k) \mathcal{P}_{\mu \nu}^{\perp}(k)+\delta^{a b} \omega_{\|}(k) \mathcal{P}_{\mu \nu}^{\|}(k) .
$$

The two projectors $\mathcal{P}^{\|}$and $\mathcal{P}^{\perp}$ are both 4-dimensionally transversal, but 3-dimensionally longitudinal and transversal, respectively [7]. We can now proceed as before and derive a closed system for the ghost form factor from its resolvent identity, assuming a bare ghost-gluon vertex at all temperatures. The result are two separate gap equations,

$$
\begin{aligned}
& \omega_{\perp}(k)=k_{0}^{2}+\mathbf{k}^{2}+\chi_{\perp}(k)+M^{2}(\beta) \\
& \omega_{\|}(k)=k_{0}^{2}+\mathbf{k}^{2}+\chi_{\|}(k)+M^{2}(\beta)+\frac{\mathbf{k}^{2}}{k_{0}^{2}+\mathbf{k}^{2}} \widetilde{M}^{2}(\beta)
\end{aligned}
$$

where the two curvature components are related to the ghost form factor by different temperaturedependent integral equations, and the two tadpole contributions are now also temperature-dependent. (For further details and explicit formulae, see Ref. [7].)

The renormalization should, in principle, be carried out at $T=0$ using the same counter terms as discussed above. The finite temperature parameters such as $M^{2}(\beta)$ etc. are then related to the $T=0$ parameters $M_{A}^{2}$ etc. through finite temperature-dependent equations [7]. For practical calculations, these equations are, however, not well suited, and it is more convenient ot fit the numbers $M^{2}(\beta)$ and $\widetilde{M}^{2}(\beta)$ directly from the lattice data at every temperature, and prescribe the wave function renormalizations as in the case $T=0$. This simplified procedure circumvents the cumbersome equations for the temperature-dependent mass parameters and effectively describes the complete set of all renormalized propagators with just 4 parameters at any $\beta$.

Numerically, the solution of the finite-temperature system is quite expensive, since we now have five profile functions of $\left(k_{0}, \mathbf{k}\right)$, which corresponds to $5 N$ scalar functions of $\mathbf{k}^{2}$, if $N$ is the maximal

\footnotetext{
${ }^{3}$ For simplicity, we continue to write the momentum argument as $k$, even though the profiles depend on $k_{0}$ and $\mathbf{k}$ separately.
} 

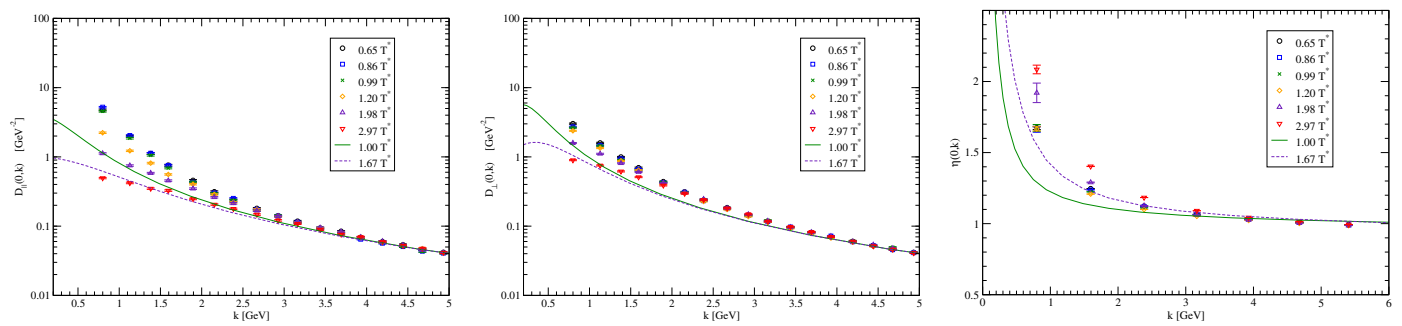

Figure 3. From left to right: the parallel and perpendicular gluon propagator and the ghost form factor at non-zero temperature, compared to high-precision lattice data [11].

number of Matsubara frequencies included in the system. All these functions are coupled in a nonlinear way, which easily results in a factor of 1000 or more in CPU time as compared to the $T=0$ case. For practical reasons, we must therefore limit the number of Matsubara modes to $N \leq 40$ which means that we cannot go down with the temperature to much lower than $T \approx T^{*}$. Still, we were able to show [7] that the finite temperature propagators at $T / T^{*}=1$ and $T / T^{*}=1.67$ exhibit all the qualitative features of the full lattice results, cf. Fig. 3:

- with increasing temperature, there is a moderate suppression of the gluon propagators in the deep infrared, and a slight enhancement of the ghost form factor;

- the temperature sensitivity is larger in the components longitudinal to the heat bath;

- all propagators are only moderately affected by temperatures well up to $T=2 T^{*}$ and, in particular, there is no qualitative change in the propagators across the deconfinment phase transition.

\section{Effective potential of the Polyakov loop}

As mentioned above, the deconfinement phase transition cannot be studied in the low-order Green's functions. Instead, a direct computation of the relevant order parameter, viz. the traced Polyakov loop $\mathrm{P}(\mathbf{x})$, is required. In Ref. [13] it has been argued that the field component $A_{0}$ in Polyakov gauge $\partial_{0} A_{0}(x)=0$ can also serve as an order parameter for confinement, because it behaves similar as the Polyakov loop,

$$
\operatorname{tr}\langle\mathrm{P}(\mathbf{x})\rangle=\operatorname{tr}\left\langle e^{-\beta A_{0}(\mathbf{x})}\right\rangle \sim \operatorname{tr} e^{-\beta\left\langle A_{0}(\mathbf{x})\right\rangle} .
$$

Here, the symbol $\sim$ means "'behaves similar as"', i.e. vanishes in the deconfined phase and does not vanish (or becomes infinite) in the confined phase. The same reasoning also carries over to the background gauge on the fluctuation field $Q_{\mu} \equiv A_{\mu}-\mathrm{a}_{\mu}$,

$$
\hat{\mathrm{d}}_{\mu} Q_{\mu} \equiv\left(\partial_{\mu} \mathbf{1}+\hat{\mathrm{a}}_{\mu}\right) Q_{\mu}=0
$$

provided that the background field $\mathrm{a}_{\mu}=\delta_{\mu 0} \mathrm{a}_{0}$ is itself in Polyakov gauge, $\partial_{0} \mathrm{a}_{0}=0$. Our goal is therefore to compute the $v e v$ of the order parameter $\left\langle A_{0}\right\rangle=\mathrm{a}_{0}^{\min }$ from the location of the minimum of the effective potential.

Technically, the transfer of the variational approach from Landau to background gauge is rather simple and amounts to the replacement of the partial by the covariant derivative, $\partial_{\mu} \rightarrow \hat{\mathrm{d}}_{\mu}$ in a few strategic places. Since the background field $\mathrm{a}_{0}$ for the present study can be taken constant, the replacement corresponds to a mere shift in the momentum arguments and we can recycle the solution 

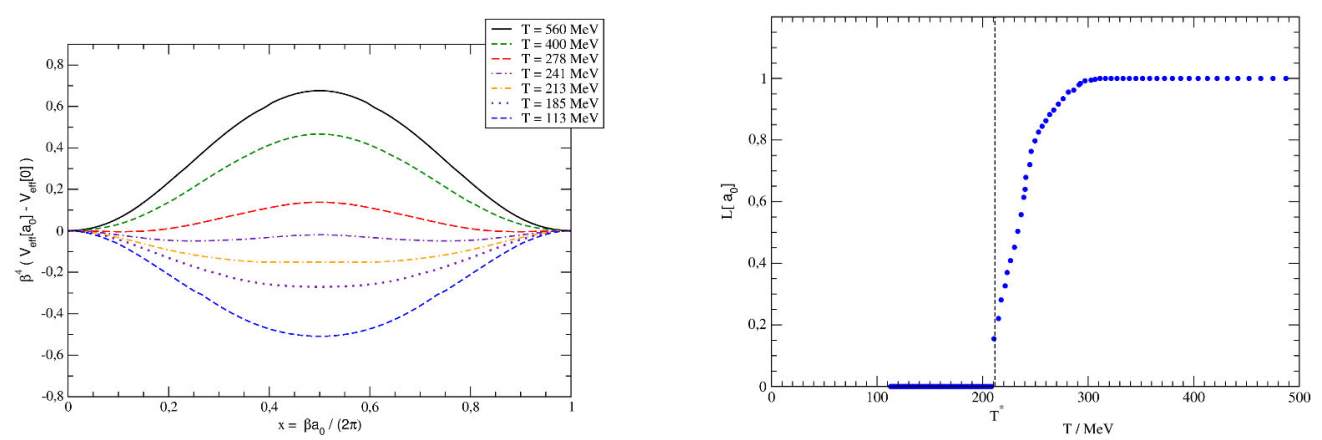

Figure 4. Left: The effective potential $V_{\text {eff }}(x)$ for the Polyakov loop on the $S U(2)$ Weyl alcove $x \in[0,1]$. Right: The Polyakov Loop $\langle\mathrm{P}\rangle$ as a function of temperature, extracted from the minimum of $V_{\text {eff }}$.

of the variational problem in Landau gauge with shifted arguments. More precisley, $\hat{\mathrm{d}}_{\mu}$ is a colour matrix and we must first go to a colour base in which $\hat{\mathrm{d}}_{\mu}$ is diagonal, $\hat{\mathrm{d}}_{\mu}^{a b}=\mathbf{e}_{\sigma}^{a}\left(\mathbf{e}_{\tau}^{b}\right)^{*} \delta^{\sigma \tau} \mathrm{d}_{\mu}^{\sigma}$. This is the so-called root decomposition of the colour algebra, and the momentum shift becomes

$$
\partial_{\mu}(p)=i p_{\mu} \rightarrow \mathrm{d}_{\mu}^{\sigma}(p)=i\left(p_{\mu}-\sigma \mathrm{a}_{0} \delta_{\mu 0}\right) \equiv i p_{\mu}^{\sigma}
$$

for every simple root vector $\sigma$. We must also replace the factor $\left(N^{2}-1\right)$ from the colour traces in Landau gauge by a sum over all simple roots.

It should be emphaiszed that this recipe only holds when using the $T=0$ kernels even at finite temperature (which is introduced as before by compactifying the euclidean time direction). The temperature dependent kernels involve the background field in other ways than just through the covariant derivative $\hat{\mathrm{d}}_{\mu}$, and the same also happens if we go beyond two-loop in the effective potential. However, as we have seen above, the kernels are only mildly affected by temperature, and it has been further argued in Ref. [13] that the dominant contributions to the integral equations come from momentum and frequency regions where the finite temperature corrections to the kernels are negligable. We will thus use the $T=0$ decoupling solutions introduced earlier as input of the present calculation.

After renormalization, the effective potential $V_{\text {eff }}$ of the Polyakov loop is obtained as the difference of the effective actions $\Gamma\left[\mathrm{a}_{0}\right]-\Gamma[0]$ per unit 4-volume. Due to global colour invariance, the potential can only depend on the eigenvalues of $\mathrm{a}_{0}=\mathrm{a}_{0}^{c} T^{c}$, which can therefore be restricted to the Cartan subalgebra from the outset. For $G=S U(2)$, there is only a single Cartan generator $T^{3}=\sigma^{3} /(2 i)$ and the fundamental domain (Weyl alcove) for the background $\mathrm{a}_{0}$ is conveniently parametrized by the rescaled component $x \equiv \beta \mathrm{a}_{0}^{3} / 2 \pi \in[0,1]$. The explicit formula for $V_{\text {eff }}(x)$ can be put in the form

$$
\beta^{4} V_{\mathrm{eff}}(x)=\beta^{4} W(x)+\frac{6}{\pi^{2}} \sum_{m=1}^{\infty} \frac{1-\cos (2 \pi m x)}{m^{4}} h(\beta m),
$$

where the Weiss potential in first term is the perturbative one-loop result, $\beta W(x)=\frac{4}{3} \pi^{2} x^{2}(1-x)^{2}$. The non-perturbative correction in the second term involves the function

$$
h(\lambda)=-\frac{1}{4} \int_{0}^{\infty} d \xi \xi^{2} J_{1}(\xi)\left[\ln \left(\frac{\omega(\xi / \lambda)}{(\xi / \lambda)^{2}}\right)-\frac{\chi(\xi / \lambda)}{\omega(\xi / \lambda)}\right],
$$

where $\omega$ and $\chi$ are the $T=0$ solution of the variational problem in Landau gauge and $J_{1}(\xi)$ is a regular Bessel function. The potential $V_{\text {eff }}(x)$ is periodic outside the Weyl alcocve $x \in[0,1]$. Center 

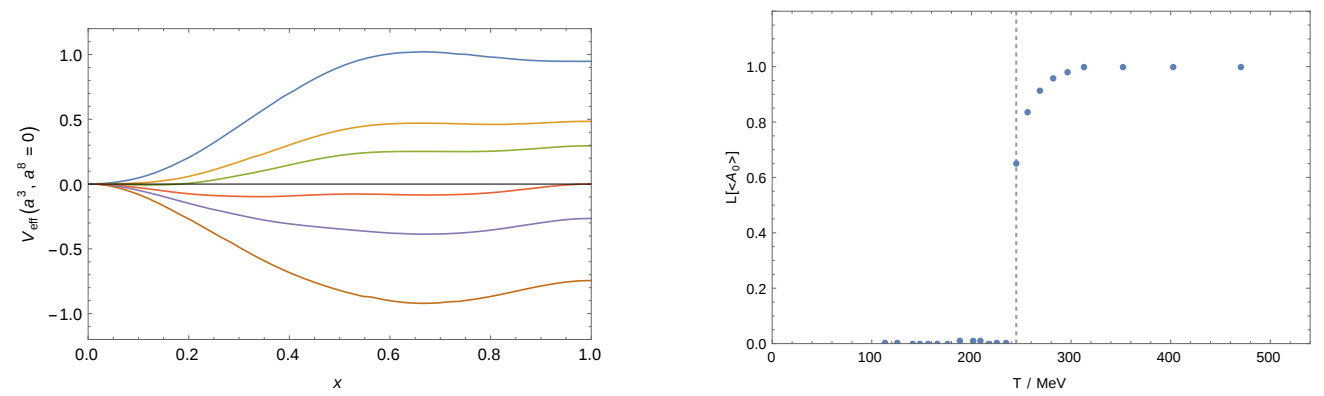

Figure 5. Left: Slices $y=0$ of the effective potential $V_{\text {eff }}(x, y)$ for the Polyakov loop on the $G=S U(3)$ Weyl alcove. Right: The Polyakov Loop $\langle\mathrm{P}\rangle$ as a function of temperature, extracted from the minimum of the effective potential.

symmetry acts as $x \rightarrow 1-x$ and the center symmetric point is hence located at $x=1 / 2$. At this point, the Polyakov loop $\mathrm{P}$ vanishes and we have confinement, while the maximally center breaking configurations with $\mathrm{P}=1$ located at $x=0$ and $x=1$ describe deconfinement.

The deconfinement phase transition thus occurs as a rapid change of the location of the minimum of $V_{\mathrm{eff}}(x)$, from $x=0$ and $x=1$ at $T>T^{*}$ to $x=1 / 2$ at $T \leq T^{*}$. This is shown in Fig. 4. The transition is clearly second order and the phase transition temperature can be translated into absolute units by using the mass scale $M_{A}^{2}$ introduced in the $T=0$ propagators. This gives a value of

$$
T^{*} \approx 214 \mathrm{MeV}
$$

which is in fair agreement with the lattice findings of $T^{*} \approx 300 \mathrm{MeV}$ [14], in particular since the determination of the scale $M_{A}^{2}$ from the fit of the variational solutions to lattice data has rather large uncertainties.

Similar results can be obtained for the colour group $G=S U(3)$, which has rank 2 so that the effective action of the Polykov loop can be parametrized by two rescaled components ${ }^{4}$

$$
x \equiv \frac{\beta \mathrm{a}_{0}^{3}}{2 \pi} \in[0,1], \quad y \equiv \frac{\beta \mathrm{a}_{0}^{8}}{2 \pi} \in\left[0, \frac{2}{\sqrt{3}}\right] .
$$

Fig. 5 shows slices $\mathrm{y}=0$ of the effective potential $V_{\text {eff }}(x, y)$ at various temperatures, and the Polyakov loop as obtained from the minimum of $V_{\text {eff }}$. The transition is now clearly first order and the transition temperature

$$
T^{*} \approx 245 \mathrm{GeV}
$$

is in good agreement with the lattice estimate of $T^{*}=284 \mathrm{MeV}$ [14]. Fig. 6 finally shows the effective potential $V_{\text {eff }}(x, y)$ for a temperature $T=141 \mathrm{MeV}$ in the confined, and $T=400 \mathrm{MeV}$ in the deconfined phase. As one can clearly see, the minimum moves from the three center symmetric points to the center breaking points in the corner of the Weyl alcove as we cross the phase transition.

\section{Conclusions}

In this talk, I have argued that the covariant variational approach to gauge theory can be used to describe the low-order Green's functions rather accurately, both at zero and non-zero temperature,

\footnotetext{
${ }^{4}$ This rectangular parametrization actually covers two triangular Weyl alcoves, i.e. two periods of the effective potential.
} 

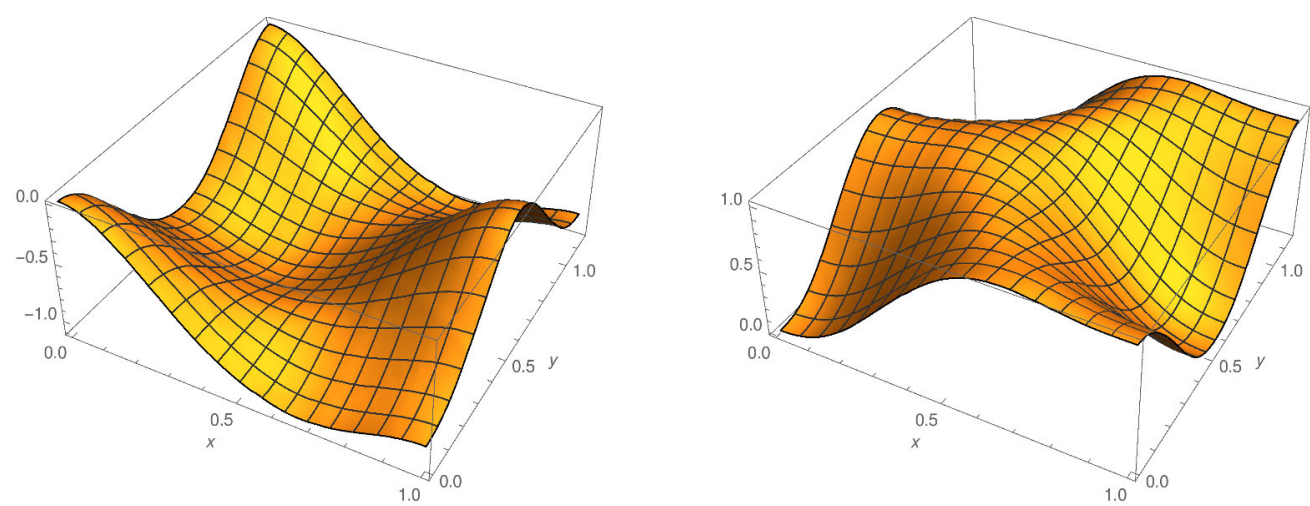

Figure 6. The effective potential $V_{\text {eff }}(x, y)$ for the SU(3) Polyakov loop, for a temperature $T=141 \mathrm{MeV}$ (left), and $T=400 \mathrm{MeV}($ right $)$.

even when based on a rather simple Gaussian ansatz. The system can be renormalized conventionally through local counter terms and is well amenable to numerical treatment. The deconfinement phase transition can be studied from the effective action of the Polyakov loop. We find the correct orders and qualitative behaviour of the transition for both colour groups $\mathrm{SU}(2)$ and $\mathrm{SU}(3)$, and numerical values for the transition temperatures which are in good agreement with lattice data.

One immediate future application is the study of thermodynamics, as the pressure of the YangMills system is readily accessable from the free or effective action studied for the Polyakov loop. In addition, we also plan to extend the variational ansatz beyond Gaussian measures, and employ DysonSchwinger equations to obtain the necessary moments. Finally, the inclusion of dynamical fermions and the description of full QCD is currently underway.

\section{References}

[1] J.M. Pawlowski, Annals Phys. 322, 2831 (2007); H. Gies, Lect. Notes Phys. 852, 287 (2012).

[2] R. Alkofer and L. von Smekal, Phys. Rept. 353, 281 (2001); C. S. Fischer, J. Phys. G32, R253 (2006); D. Binosi and J. Papavassiliou, Phys. Rept. 353, 1 (2009).

[3] M. Tissier and N. Wschebor, Phys. Rev. D82, 101701 (2010).

[4] D. Zwanziger, Nucl. Phys. B399, 477 (1993).

[5] C. Feuchter and H. Reinhardt, Phys. Rev. 70, 105021 (2004);

C. Feuchter and H. Reinhardt, arXiv:hep-th/0402106 (2004).

[6] M. Quandt, H. Reinhardt and J. Heffner, Phys. Rev. 89, 065037 (2014).

[7] M. Quandt and H. Reinhardt, Phys. Rev. D92, 025051 (2015).

[8] M. Quandt and H. Reinhardt, Phys. Rev. D94, 065015 (2016).

[9] J.C. Taylor, Nucl. Phys. B33, 436 (1971).

[10] A. Maas, Phys. Rev. D93, 054504 (2016)

[11] I. Bogolubsky et al., Phys. Lett. B676, 69 (2009).

[12] C.W. Bernard, Phys. Rev. D9, 3312 (1974).

[13] J. Braun, H. Gies and J. M. Pawlowski, Phys. Lett. B684, 262 (2010).

[14] B.Lucini, M.Teper and U.Wenger, J. High Energy Phys. 01, 061 (2004). 